\title{
Surveillances croisées et rivalité gréco-turque en Thrace occidentale : entre coercition et contrôle social
}

Cross monitoring and Greek-Turkish rivalry in Western Thrace: between coercion and social control

Jeanne Hersant

\section{OpenEdition}

Journals

Édition électronique

URL : http://journals.openedition.org/ejts/2693

DOI : 10.4000/ejts.2693

ISSN : $1773-0546$

Éditeur

EJTS

Référence électronique

Jeanne Hersant, « Surveillances croisées et rivalité gréco-turque en Thrace occidentale : entre coercition et contrôle social », European Journal of Turkish Studies [En ligne], 8 | 2008, mis en ligne le 24 mars 2015, consulté le 16 février 2020. URL : http://journals.openedition.org/ejts/2693; DOI : $10.4000 /$ ejts. 2693 


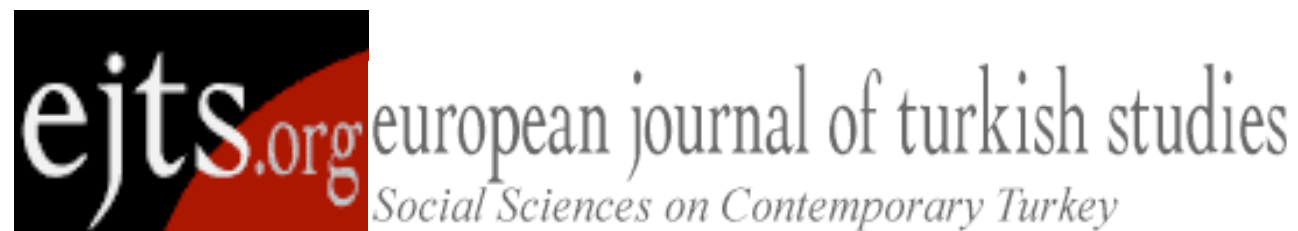

Hersant, Jeanne (2008) 'Surveillances croisées et rivalité gréco-turque en Thrace occidentale : entre coercition et contrôle social', European Journal of Turkish Studies, Thematic Issue $N^{\circ} 8$, No. 8 | Surveiller, normaliser, réprimer, URL : http://www.ejts.org/document2693.html

To quote a passage, use paragraph (§).

Surveillances croisées et rivalité gréco-turque en Thrace occidentale : entre coercition et contrôle social

Jeanne Hersant

\begin{abstract}
This article examines the construction of a Turkish minority in Western Thrace from a historical perspective. It suggests that the identification of Turks in Thrace as a minority is due, first, to the fact that Greek authorities impeded the economic development of the region and its Muslim minority, and, second, to the Turkish state's promotion of Turkish national identity among this minority. In other words, this article deals with two concurrent state policies in Western Thrace, with particular attention to both the Greek and Turkish authorities' changing techniques of surveillance (among the Muslim population) following the warmer relations between the two countries that began at the end of the 1990s.
\end{abstract}


Hersant, Jeanne (2008) 'Surveillances croisées et rivalité gréco-turque en Thrace occidentale : entre coercition et contrôle social', European Journal of Turkish Studies, Thematic Issue N 8 , No. 8 | Surveiller, normaliser, réprimer, URL : http://www.ejts.org/document2693.html

To quote a passage, use paragraph (§).

\section{Mise en contexte}

Ce texte s'intéresse à deux logiques étatiques concurrentes en Thrace occidentale. La constitution d'une minorité turque en Thrace - en tant que réalité sociologique et entité politique ${ }^{1}$ est historiquement tributaire d'un double processus de particularisation de la région et de sa 'minorité musulmane' par les autorités grecques d'une part, et d'autre part de la diffusion d'un sentiment national turc au sein de cette minoritée. Désignée comme 'musulmane' dans le traité de Lausanne, cette minorité était de facto reconnue comme turque jusqu'aux années 1960, jusqu'à ce que le contentieux chypriote fasse craindre l'irrédentisme turc aux autorités grecques. Aujourd'hui, la dénomination de ce groupe est l'objet d'une lutte symbolique entre ses représentants et l'État turc d'une part, qui revendiquent la turcité de la minorité, et l'État grec de l'autre, qui promeut quant à lui l'expression d'une identité pomaque 3 . Puisqu'il convient d'éviter que les catégories qui s'affrontent deviennent celles du chercheur, nous parlerons en général de population musulmane, en usant de guillemets lorsqu'il sera question de la dénomination officielle grecque de 'minorité musulmane'.

[2] D'autre part, nous aurons recours aux caractères en italique lorsqu'il sera question de Turcs, en référence aux acteurs et promoteurs du mouvement identitaire, engagés depuis les années 1980 dans une mobilisation politique visant à faire reconnaître comme turque l'ensemble de la

${ }^{1}$ La notion d'entité politique vise d'une part à rendre compte du travail des leaders politiques turcs de mise sur agenda localement d'enjeux et de revendications spécifiques au groupe défini comme turc (à l'exclusion de projets concernant l'ensemble de la société locale). D'autre part, il s'agit d'illustrer la façon dont - pendant les élections locales tout au moins - les acteurs et partis politiques grecs centrent en grande partie leur discours et leur campagne sur ce groupe; on pourrait également utiliser l'expression de 'groupe de référence' forgée dans les études sur les partis politiques. Voir sur ce point Leveau (1991).

${ }^{2}$ Il s'agit de celle qui fut exemptée de l'échange obligatoire de population avec la Turquie en 1924. Cet échange fut défini par un protocole additionnel au traité de Lausanne, signé après la défaite de l'armée grecque à Izmir (1922). II délimitait les frontières de l'actuelle Turquie et, révisant le traité de Sèvres de 1920, mettait fin aux ambitions européennes sur le territoire de l'ancien Empire ottoman. ${ }^{3}$ La minorité musulmane est conventionnellement présentée, dans les différents travaux ou écrits journalistiques qui se multiplient depuis les années 1990, comme étant constituée de Turcs (turcophones), Tziganes (turcophones) et Pomaques (ou Pomak, locuteurs d'un dialecte du bulgare). II semble pourtant que les autorités grecques n'utilisent ce dernier terme que depuis les années 1970, et qu'il n'était pas d'usage courant au sein de la minorité jusqu'à l'exode rural des années 1990 (Demetriou 2002). 
Hersant, Jeanne (2008) 'Surveillances croisées et rivalité gréco-turque en Thrace occidentale : entre coercition et contrôle social', European Journal of Turkish Studies, Thematic Issue N 8 , No. 8 | Surveiller, normaliser, réprimer, URL : http://www.ejts.org/document2693.html

To quote a passage, use paragraph (§).

minorité par l'Etat grec et les instances européennes ${ }^{4}$. Ce procédé permet en outre de les distinguer des Turcs citoyens de la République de Turquie. Les enquêtes ayant donné lieu à ce travail ont été réalisées entre août 2002 et juillet 2005 dans différentes localités des départements de Xanthi et du Rhodope5, ainsi qu'en Turquie, principalement à Istanbul6. Elles sont complétées par une étude de la presse turcophone locale, à travers le dépouillement de l'hebdomadaire Gündem (Ordre du jour) entre août 2002 et septembre 2007.

[3] Cet hebdomadaire creé en 1995 et publié à Komotini illustre la professionnalisation du journalisme en Thrace occidentale: ses journalistes sont les premiers à avoir suivi une formation universitaire spécialisée. II comptait en 2007 trois journalistes salariés à temps plein, dont la rédactrice en chef. Deux d'entre eux ont eu une première expérience professionnelle au sein de grands médias (presse et audiovisuel) en Turquie, et une expérience militante au sein des associations de Turcs de Thrace occidentale, en Turquie et en Grande-Bretagne. Ils parlent le grec et l'anglais couramment, ils incarnent à ce titre la nouvelle élite sur laquelle les autorités turques souhaitent s'appuyer pour assurer la promotion d'une identité turque dans la région?.

${ }^{4}$ Ce procédé est inspiré du travail de Charles Suaud (1997), qui distingue la Vendée en tant que territoire géographique et entité administrative de la Vendée en tant que territoire investi d'imaginaires réfractaires à l'État.

${ }^{5}$ La Thrace occidentale 'historique' (en référence à l'ancienne organisation administrative grecque) est composée d'un troisième département, l'Evros, frontalier avec la Turquie, dont les musulmans ont été 'invités' à partir avant l'échange de population de 1924. Depuis 1994, la Thrace fait partie de la région (periphereia) Macédoine orientale-Thrace occidentale, qui compte cinq départements. Les musulmans seraient aujourd'hui entre 100 et 120000 et représenteraient la moitié de la population totale des départements du Rhodope et de Xanthi (Dalègre 1997), mais ce chiffre n'est qu'une estimation : les critères de langue et de religion n'apparaissent plus dans les statistiques grecques depuis 1951.

${ }^{6}$ Des entretiens formels ou informels, individuels ou dans un cadre familial, ont été réalisés avec 120 personnes, en Grèce, Allemagne et Turquie. Une quarantaine d'entre elles ont été rencontrées à plusieurs reprises, souvent dans deux pays ; une vingtaine étaient des interlocuteurs de référence, avec qui des contacts réguliers ont été établis, et qui m'ont introduite dans leur cercle de sociabilité.

${ }^{7}$ Gündem est subventionné par le consulat turc comme d'autres titres de la presse turcophone bien que cela ne soit jamais admis. A l'époque de ces enquêtes, seuls les journaux venant de Turquie (Milliyet et Hürriyet) sont distribués en kiosque ; Gündem est commercialisé grâce à des circuits de distribution parallèles, des commerces servant de dépôt, ou par abonnement. On peut donc s'interroger sur la viabilité économique du journal: il comptait en 2003 à peine une centaine d'abonnés (particuliers ou associations) à l'étranger (Allemagne, Turquie, Grande-Bretagne), et nous n'avons pu obtenir le nombre des abonnés en Thrace. Depuis 2006, Gündem est disponible sur internet, ce qui simplifie le problème de sa diffusion. 
Hersant, Jeanne (2008) 'Surveillances croisées et rivalité gréco-turque en Thrace occidentale : entre coercition et contrôle social', European Journal of Turkish Studies, Thematic Issue N 8 , No. 8 | Surveiller, normaliser, réprimer, URL : http://www.ejts.org/document2693.html

To quote a passage, use paragraph (§).

\section{Pour une sociologie des institutions en Thrace occidentale}

[4] Le consulat général de Turquie qui se trouvait à Kavala aurait été transféré à Komotini en 1923, au moment de l'échange de population (Özgüç 1974 : 160). C'est aujourd'hui l'un des plus importants consulats turcs au monde en termes de moyens financiers ${ }^{9}$, alors qu'il n'y a pas de ressortissants turcs dans la région. La raison en est le statut de 'territoire perdu' de la Thrace occidentale dans le nationalisme turc tel que diffusé par les canaux officiels ${ }^{10}$, mais aussi le statut d'État garant reconnu à la Turquie par le traité de Lausanne ${ }^{11}$. 'Nous avons des droits ici, en vertu du traité de Lausanne', tel fut le commentaire d'un consul général de Turquie en poste à Komotini lors d'un entretien. Pourtant, le consul intervient dans toute une série d'actes administratifs et de consultations politiques dont la portée dépasse d'une part les dispositions de ce traité, et d'autre part les attributions traditionnelles d'une représentation consulaire. C'est l'interlocuteur des autorités grecques pour diverses formalités administratives, en tant qu'institution tutélaire des membres de la 'minorité musulmane'. En effet, bien que citoyens grecs, les membres de cette minorité sont sous la tutelle du ministère grec des Affaires étrangères.

[5] La formation de la scène politique en Thrace occidentale, dans les années 1930, reflète une logique interétatique en vertu de laquelle des prérogatives sont reconnues à l'État turc. Hormis les accords négociés entre les deux Etats, la présence à Komotini d'un Consulat général de Turquie a joué un rôle non négligeable sur l'organisation sociale et politique de la population musulmane, notamment autour d'associations imitées de celles qui voyaient alors le jour dans la Turquie kémaliste. Ces institutions ont permis la transposition des enjeux et luttes politiques de la République

${ }^{8}$ Sevasti Trubeta (1999: 83) affirme quant à elle que le consulat général de Turquie, initialement situé à Thessalonique (où s'en trouve un autre aujourd'hui), fut déplacé à Komotini à la suite de la signature du traité d'amitié entre Mustafa Kemal et Eleftherios Venizelos, en 1930.

${ }^{9}$ Entretien avec un ancien journaliste de la presse locale turcophone, janvier 2002.

${ }^{10}$ La Thrace occidentale fait partie des 'territoires perdus' inclus dans le Pacte National (Misak-I Milli), adopté sous l'égide de Mustafa Kemal par la première Assemblée Nationale d'Ankara, en 1920. Cette assemblée ne reconnaissait pas la cession des territoires entérinée par le traité de Sèvres, négocié par le gouvernement ottoman d'Istanbul.

${ }^{11}$ Comme à plusieurs autres pays, dont la France, l'Angleterre et le Japon. La prééminence de la Turquie s'est affirmée à travers plusieurs accords bilatéraux consacrant par exemple l'enseignement du turc comme langue commune aux musulmans dans les écoles de la minorité (Hersant 2007). Cet aspect relevant d'une logique de coopération interétatique, il ne rentre pas dans le cadre de la cogouvernementalité en tant que tel, et ne sera pas traité ici. 
Hersant, Jeanne (2008) 'Surveillances croisées et rivalité gréco-turque en Thrace occidentale : entre coercition et contrôle social', European Journal of Turkish Studies, Thematic Issue N 8 , No. 8 | Surveiller, normaliser, réprimer, URL : http://www.ejts.org/document2693.html

To quote a passage, use paragraph (§).

turque en Thrace occidentale ; elles ont cristallisé les allégeances et partis pris politiques au sein de 'la minorité musulmane' pendant plusieurs décennies, notamment le conflit entre partisans des réformes kémalistes et défenseurs de l'héritage ottoman, perceptible jusqu'aux années 1970.

[6] Comme en contrepartie des importantes prérogatives accordées localement à l'État turc, les institutions de l'État grec ont mis en œuvre, depuis les années 1930, une gestion sécuritaire de la Thrace occidentale et de sa population musulmane, à travers une politique de sécurisation des frontières. Cette politique a abouti plus tard, dans le contexte de la guerre froide puis du conflit chypriote, et surtout dans la période plus récente, à la création d'un front intérieur. Tout le nord de la Grèce fut, dans son périmètre frontalier, une zone militaire fermée de 1936 à la fin des années 1990. Cette mesure fut assortie en Thrace occidentale de la mise en place d'une administration parallèle, sous tutelle militaire. C'est ainsi que s'est constitué le 'stigmate' auquel fait allusion Pierre Bourdieu dans son analyse des constructions identitaires régionales et ethniques (Bourdieu $1980: 69-70$ ).

[7] Le processus de constitution d'une minorité est également corollaire d'une assignation identitaire turque, et lié à la prise en charge des membres du groupe par les institutions qui représentent l'État turc en Thrace occidentale, ce terme étant compris au sens que lui donne Jacques Lagroye, comme 'un ensemble de pratiques qui ont quelque rapport - parfois manifeste, parfois ténu - avec l'existence de relations réglées et entretenues qu'on appelle généralement pour cela des institutions' (Lagroye 2002: 115). II s'agit en somme ici de s'intéresser aux pratiques sociales qui 'font exister' les institutions dans leur acception conventionnelle, ainsi qu'aux personnes qui les incarnent. À la fois représentant de l'Etat turc et notable local, le consul en poste à Komotini joue un rôle essentiel dans le projet de mise en forme du corps social constitué par la population musulmane. Or, cette fonction sociale du consul est rarement prise en compte en tant que telle, l'observation microsociologique du terrain interétatique étant souvent délaissée ${ }^{12}$.

[8] La prise en compte des usages sociaux de l'institution, et de la façon dont celle-ci interagit avec ses usagers, à travers les acteurs qui la représentent ou l'incarnent, permet de dépasser une approche en termes d'affrontement ou de coopération interétatique, propre au domaine des relations internationales. Cela permet, en effet, de comprendre quand et comment ce que nous avons nommé plus haut la logique de coopération interétatique, matérialisée par une série

${ }^{12}$ On pourra néanmoins se reporter à Clayer (2007), qui a entrepris une démarche de ce type dans l'étude de la formation du nationalisme albanais. 
Hersant, Jeanne (2008) 'Surveillances croisées et rivalité gréco-turque en Thrace occidentale : entre coercition et contrôle social', European Journal of Turkish Studies, Thematic Issue N 8 , No. 8 | Surveiller, normaliser, réprimer, URL : http://www.ejts.org/document2693.html

To quote a passage, use paragraph (§).

d'accords bilatéraux, a fait place à de la co-gouvernementalité. Par ce terme employé en référence à Michel Foucault, nous entendons un partage concernant les prérogatives relatives à la gestion de la 'minorité musulmane', étrangère au corps national grec tel que défini officiellement ; c'est aussi une concurrence pour l'appropriation ne serait-ce que symbolique du territoire de la Thrace et un affrontement des savoirs produits par les deux États sur une minorité dont il s'agit de 'prouver' qu'elle est tantôt 'musulmane' tantôt 'turque'13.

\section{Les mécanismes de la co-gouvernementalité}

[9] II y a tout d'abord une dimension de gestion conjointe qui se rapporte à une situation de paix, l'intégrité territoriale de la Grèce n'étant pas en jeu: il n'y a pas d'affrontement armé ni de présence militaire turque en Thrace occidentale. Tout en déléguant certaines prérogatives à l'État turc concernant la gestion (administrative, scolaire, etc.) de la 'minorité musulmane', l'État grec renforce sa souveraineté, au sens premier de contrôle territorial, par une tutelle administrative d'Athènes sur la Thrace occidentale et par une sécurisation de la zone frontalière avec la Turquie. Mais cela ne suffit pas à rendre compte de la spécificité de la configuration locale. La cogouvernementalité est une sorte de front intérieur, de 'conquête de l'Ouest' menée par un État, qui concerne une population considérée comme une 'minorité nationale' mais établie sur un territoire étranger et frontalier, qui ne saurait être annexé. Les mécanismes de la co-gouvernementalité se sont mis en place progressivement durant les deux ou trois dernières décennies, les différents éléments qui la caractérisent se combinant différemment d'une période à l'autre.

[10] De la définition de Michel Foucault (2001: 653), nous retiendrons les éléments suivants pour caractériser la co-gouvernementalité en Thrace occidentale: tout d'abord, l'aspect de la gouvernementalité visant à faire prospérer la population sur laquelle s'exerce le pouvoir d'État; ensuite les mécanismes disciplinaires. L'État turc, par l'intermédiaire de son consul général, bénéficie d'une importante dévolution de compétences, en dehors de tout cadre juridique, relatives à la gestion

${ }^{13}$ Parmi les savoirs d'Etat, des projections démographiques réalisées dans les années 1990 visent à contrer les recensements grecs (note 5), pour mettre en avant le fait 'qu'étant donné le taux de natalité de 1923 pondéré jusqu'à aujourd'hui, il devrait y avoir pas moins de 600000 Turcs en Thrace' (entretien avec l'initiateur de cette projection). A la diffusion dans les années 1980 d'écrits fondés sur des tests sanguins dans une université grecque pour prouver la 'parenté génétique' entre Grecs et Pomaques (Anastassiadou 1991) répond la diffusion d'une toponymie entièrement turque de la Thrace occidentale. Etant donnée la thématique du dossier, cet aspect ne sera pas développé ici, dans le souci de se concentrer sur les mécanismes de surveillance proprement dits. 
Hersant, Jeanne (2008) 'Surveillances croisées et rivalité gréco-turque en Thrace occidentale : entre coercition et contrôle social', European Journal of Turkish Studies, Thematic Issue N 8 , No. 8 | Surveiller, normaliser, réprimer, URL : http://www.ejts.org/document2693.html

To quote a passage, use paragraph (§).

administrative ou culturelle de la 'minorité musulmane', qui sont en théorie du ressort des autorités grecques; tant sur le plan de la redistribution économique que par la diffusion d'une identité nationale, d'abord au sens premier de recensement des nationaux par la distribution de cartes d'identité). Ainsi, sur la base des 'certificats d'établis' rédigés en français et distribués en 1930 par une Commission mixte internationale aux populations exemptées de l'échange de population entre la Grèce et la Turquie, le consulat général de Turquie délivre aujourd'hui des certificats du même nom (etabli belgesi) aux musulmans, qui leurs procurent de facto en Turquie la plupart des droits économiques et sociaux dont jouissent les ressortissants turcs. La diffusion d'un sentiment national passe également par des rituels à l'occasion de la célébration des fêtes nationales turques, auxquels sont associés les élèves des écoles de la minorité.

[11] Ainsi, non seulement l'État grec interdisait aux musulmans, jusqu'aux années 1990, d'accéder aux emplois publics civils et militaires ${ }^{14}$, mais il a en plus délégué aux autorités turques le soin de rémunérer ceux de la minorité qui, statutairement, relevaient de la fonction publique mais en étaient exclus : personnel religieux (muftis réfractaires ${ }^{15}$, imams, muezzin) et enseignants des écoles de la minorité16. En somme, les opportunités de promotion sociale offertes aux musulmans, au moins jusqu'à ces dernières années, l'ont été par l'intermédiaire du consulat, qu'il s'agisse de trouver un emploi en Thrace ou de partir étudier en Turquie. À cela s'ajoutent les subventions à la presse locale et la rémunération du personnel associatif ou des enseignants au chômage.

[12] La co-gouvernementalité s'exprime aussi dans les mécanismes disciplinaires, sur lesquels se concentreront les développements à suivre. En dehors de l'acte législatif 'qui pose la loi' et de l'acte judiciaire 'qui punit le coupable', les mécanismes disciplinaires supposent 'toute une série de techniques adjacentes, policières, médicales, psychologiques, qui relèvent de la surveillance, du diagnostic, de la transformation éventuelle des individus' (Foucault 2004 : 7). Dans le cas présent, la

${ }^{14}$ C'est seulement vers 1965 que les musulmans ont été incorporés à l'armée grecque en tant qu'appelés du contingent ; en revanche, jusqu'aux années 1990 le grade d'officier leur était interdit.

${ }^{15}$ II s'agit des muftis qui ne reconnaissent pas la procédure d'appointement des autorités grecques, et qui se sont imposés comme leaders du mouvement identitaire turc à la fin des années 1980 face aux deux muftis réguliers qui eux sont fonctionnaires de l'Etat grec.

${ }^{16}$ Il s'agit là des écoles primaires telles que prévues par le traité de Lausanne, dont les enseignants sont initialement rémunérés par les membres de la minorité : les deux lycées de la minorité ont été créés après le traité de Lausanne $(1952,1968)$ et leurs enseignants sont des fonctionnaires grecs presque jamais recrutés parmi les musulmans. Pour plus de précisions, Hersant (2007). 
Hersant, Jeanne (2008) 'Surveillances croisées et rivalité gréco-turque en Thrace occidentale : entre coercition et contrôle social', European Journal of Turkish Studies, Thematic Issue N 8 , No. 8 | Surveiller, normaliser, réprimer, URL : http://www.ejts.org/document2693.html

To quote a passage, use paragraph (§).

discipline désigne le fait de quadriller une population en lui donnant le sentiment d'être constamment sous surveillance. En effet, si la définition de la gouvernementalité de Michel Foucault ne mentionne pas explicitement la discipline mais plutôt les dispositifs de sécurité, celui-ci associe néanmoins l'aspect disciplinaire et l'aspect régularisateur des techniques de gouvernement modernes ${ }^{17}$.

[13] En tant que tel, la co-gouvernementalité est un phénomène qui s'applique surtout à la période récente ; l'ouverture politique depuis la fin des années 1990 ayant permis un partage des compétences et une délimitation des aires d'influence sur un mode pacifié, bien que les logiques d'inimitié soient au fond encore très prégnantes ${ }^{18}$. Ce sont les mécanismes de surveillance que nous allons étudier ici, dont la transformation illustre et accompagne l'ouverture politique et la transition d'une logique d'affrontement dans les années 1970 à 1990 à une logique 'd'amitié' interétatique perceptible dans les relations entre les représentants locaux des deux Etats.

\section{Quadrillage de la population musulmane dans le cadre du conflit chypriote (1970-2000)}

[14] Une logique d'affrontement prévaut entre la Grèce et la Turquie, de la fin des années 1960 à la fin des années 1970, au sujet de Chypre. Quand bien même la Thrace occidentale n'est pas le lieu de cet affrontement armé, elle en est d'une certaine façon le théâtre: une série d'interdictions et de mesures d'urgence ont frappé les musulmans (couvre-feu, rafles policières, etc.) immédiatement après l'annonce de l'arrivée de l'armée turque dans le nord de Chypre, en août 1974. C'est dans ce contexte que s'est mis en place un système croisé de contrôle de la population musulmane, perceptible jusqu'à la fin des années 1990.

${ }^{17}$ Par exemple, les mécanismes disciplinaires supposent 'toute une série de surveillance, contrôles, regards, quadrillages divers qui permettent de repérer, avant même que le voleur ait volé, s'il ne va pas voler'; le mécanisme régularisateur lié aux dispositifs de sécurité 'va être commandé par une série de question du genre suivant [...] : Comment statistiquement est-ce qu'on peut prévoir qu'il y aura telle ou telle quantité de vols à un moment donné, dans une société donnée, dans une ville donnée [...], dans telle couche sociale ?' (Foucault $2004: 6$ ).

${ }^{18}$ La détente politique vis-à-vis des 'musulmans' est à mettre en rapport avec le processus d'européanisation de la Grèce (Hersant 2001 et 2007). Quant à la détente gréco-turque, il nous paraît hasardeux de l'imputer au mouvement de solidarité né des séismes successifs d'lzmit et Athènes en 1999 mais nous disposons de peu d'études sur le sujet ; voir par exemple Gilles Bertrand (2004). 
Hersant, Jeanne (2008) 'Surveillances croisées et rivalité gréco-turque en Thrace occidentale : entre coercition et contrôle social', European Journal of Turkish Studies, Thematic Issue N 8 , No. 8 | Surveiller, normaliser, réprimer, URL : http://www.ejts.org/document2693.html

To quote a passage, use paragraph (§).

\section{Surveillance grecque : policiers en civil et réseau d'informateurs}

[15] Côté grec, ce quadrillage opérait par le biais de policiers en civil ; ils sont la risée de nos interlocuteurs, qui s'approprient ainsi des rapports interindividuels anxiogènes pour en faire un sujet de plaisanterie, voire de canular :

Lorsqu'il était étudiant à Istanbul, Taner avait fondé avec quelques camarades une association d'étudiants turcs originaires de la Thrace. Ils ont organisé un festival cinq étés durant, dans différents villages de la région : il s'agissait de promouvoir la musique, les chansons et danses 'traditionnelles'. Lors des deux premières éditions, en 1992 et 1993, les autorités locales auraient fait des tentatives d'intimidation; des policiers en civil se seraient rendus chez sa mère pour mener une enquête. Taner dit avoir alors écrit et diffusé un article en anglais qu'il a présenté comme venant du New York Times, relatant le festival en termes élogieux. La supercherie semble grossière mais il affirme ne plus avoir été ennuyé. Selon lui les autorités grecques sont tellement soucieuses de ne plus se faire 'épingler' par les ONG européennes pour leur comportement vis-à-vis des musulmans, que n'importe quelle publicité étrangère est un gage de sécurité pour les acteurs associatifs locaux ${ }^{19}$.

Ali relata quant à lui une anecdote pour railler le 'manque de discrétion' des membres de la police 'censée être secrète', à propos de la surveillance dont fait l'objet son père, qui fait partie du personnel religieux rémunéré par le consulat turc ${ }^{20}$. Un soir de l'été 1999, alors qu'il dînait dans un restaurant de Xanthi avec son père, deux hommes sont entrés ensemble dans l'établissement 'mais se sont assis seuls à deux tables différentes' ; à la fin de la soirée, il a vu ces deux hommes quitter séparément le restaurant mais repartir dans la même voiture, preuve supplémentaire, selon lui, de leur stupidité $^{21}$.

[16] En référence aux années 1980, il est souvent fait allusion par nos interlocuteurs ${ }^{22}$, aux systèmes concurrents de rémunération d'intermédiaires, nommés 'espions' ou 'agents' par l'un ou l'autre 'camp', voire par les deux. Cette surveillance a suffisamment marqué l'imaginaire pour ne pas être omis, laissant planer le souvenir, réel ou fantasmé, d'un climat de délation généralisée. La surveillance des autorités grecques s'appuyait en outre sur un réseau d'informateurs parmi les musulmans, qui auraient eu pour rôle de les tenir informées des allées et venues des personnes

${ }^{19}$ Entretien réalisé en janvier 2002.

${ }^{20}$ Jusqu'aux années 1990, des policiers en civil suivaient systématiquement les déplacements des personnalités turques mais aussi des étrangers de passage dans la région (Dalègre 1997).

${ }^{21}$ Entretien réalisé en février 2002.

${ }^{22}$ Vemund Aarbakke (2000) détaille également cet aspect, mais en privilégiant l'anecdote (avérée ou rapportée) au détriment de l'analyse sociologique. 
Hersant, Jeanne (2008) 'Surveillances croisées et rivalité gréco-turque en Thrace occidentale : entre coercition et contrôle social', European Journal of Turkish Studies, Thematic Issue N 8 , No. 8 | Surveiller, normaliser, réprimer, URL : http://www.ejts.org/document2693.html

To quote a passage, use paragraph (§).

parties en Turquie, qui se voyaient alors déchues de la nationalité grecque au titre de l'article $19 \mathrm{du}$ Code de la nationalitée ${ }^{23}$.

[17] Un témoignage obtenu indirectement suggère par ailleurs que, même si les emplois du service public en Grèce s'ouvrent aujourd'hui aux musulmans, les critères de recrutement s'appuient sur des loyautés datant de cette époque révolue. Une amie de lycée de l'une de nos interlocutrices régulières a été embauchée comme agent contractuel à la mairie de Xanthi en 2003. Lors de son entrée en fonction, on lui aurait suggéré qu'elle avait été choisie pour le poste en signe de reconnaissance, car son patronyme était connu des autorités locales. La jeune femme s'était alors rappelée d'allusions au sujet de son grand-père, entendues lorsqu'elle était enfant, qu'elle avait prises pour des commérages (dedikodu) sans autre fondement que la malveillance ${ }^{24}$.

[18] Même si le sujet est rarement approfondi, les témoignages les plus elliptiques sont chargés de sens. Ce quadrillage de la population, a laissé des traces perceptibles à travers la méfiance diffuse que ressentent à se raconter ceux de mes interlocuteurs qui ont quitté la Thrace pour la Turquie après la crise de Chypre $^{25}$, les remords qu'ils ont parfois après l'avoir fait, et le sentiment d'une menace de représailles, de punition (par exemple l'interdiction de retourner en Grèce) qui pèserait sur eux-mêmes ou leur famille. Ce climat affleurait parfois des conversations menées en Turquie par des non dits, demi silences, regards échangés et conversations brusquement écourtées ou déviées. Ces craintes contrastaient avec la liberté de ton de nos interlocuteurs en Thrace qui, eux, ont vécu la transition vers un système politique ouvert depuis la fin des années 1990. En Thrace comme en Turquie, néanmoins, il est arrivé à plusieurs reprises qu'une personne

${ }^{23}$ Cette disposition prévoyait que tout ressortissant hellénique 'd'origine non grecque' pouvait être déchu de sa nationalité s'il quittait le pays 'sans intention de retour', et pour y mener des activités dommageables pour la nation. Le fait d'émigrer, d'étudier ou simplement de voyager en Turquie entrait dans ce type d'activité et cet article a été principalement utilisé à l'encontre de la 'minorité musulmane' entre 1955 et 1998, date à laquelle le Parlement grec l'a abrogé, suivant une injonction du Conseil de l'Europe et du Parlement européen (Hersant 2001).

${ }^{24}$ Entretien réalisé en juin 2004.

${ }^{25}$ Officiellement il n'y a plus d'émigration de la Grèce vers la Turquie depuis les années 1960, la majorité des flux ont dès lors été clandestins. Dans les années 1950 la bourgeoisie libérale a été incitée au départ (Labbé 2000) ; la plupart de nos interlocuteurs sont des agriculteurs, instituteurs ou petits commerçants partis en Turquie ou en Allemagne pour y chercher une vie meilleure. Région sous-développée dans les années 1980, la Thrace occidentale était la plus pauvre de l'Union européenne jusqu'à l'élargissement de 2004. Sur les flux migratoires de la Thrace vers l'Allemagne et la Turquie, voir Hersant $(2005,2009)$ 
Hersant, Jeanne (2008) 'Surveillances croisées et rivalité gréco-turque en Thrace occidentale : entre coercition et contrôle social', European Journal of Turkish Studies, Thematic Issue N 8 , No. 8 | Surveiller, normaliser, réprimer, URL : http://www.ejts.org/document2693.html

To quote a passage, use paragraph (§).

qui s'était lancée en notre présence dans une diatribe contre les autorités grecques ou l'ingérence turque soit rappelée à l'ordre plus ou moins sévèrement : 'tu parles trop !' ou 'moins fort, les voisins pourraient t'entendre'.

\section{La 'liste noire' du consulat général de Turquie}

[19] Si nos interlocuteurs turcs dénoncent généralement les intermédiaires rémunérés par les autorités grecques, ils sont peu nombreux à évoquer le réseau d'informateurs entretenus par le consulat général de Turquie, grâce auquel était établie une liste noire qui fonctionnait comme un mode de régulation de la contestation, tant de l'État turc que de la vie politique locale. Jusqu'aux années 1990, le rôle du consulat général de Turquie était ouvertement coercitif : les personnalités turques qui critiquaient les positions, voire l'ingérence de la Turquie dans la région, subissaient des pressions directement ou à travers leurs enfants étudiant en Turquie ${ }^{26}$. Cette liste noire permettait de menacer toute personne mettant en cause tant la légitimité de l'interventionnisme des autorités turques dans la région, que les compétences des élus locaux qui affichaient leur allégeance au consulat turc. Elle aurait été établie dans les années suivant le coup d'État du 12 septembre, et visait ceux qui émettaient des critiques à l'encontre de la politique turque en Thrace, par exemple les propriétaires de certaines gazettes turcophones, qui étaient non seulement interdits de séjour, mais en outre menacés de voir se faire expulser leurs enfants étudiants en Turquie (Aarbakke 2000 : 380387). La menace était lourde dans la mesure où l'ascension sociale passait alors quasi exclusivement par un parcours scolaire et universitaire en Turquie.

[20] Les journalistes et propriétaires de journaux (qui en sont aussi les rédacteurs en chef) ont en effet joué un rôle prépondérant dans la vie politique locale depuis les années 1920 : la presse locale a longtemps été principalement la tribune des notables locaux. La menace pesait aussi sur ceux qui avaient des engagements politiques jugés non conformes. C'est le cas de Kemal, sympathisant d'extrême gauche et initiateur, au début des années 1980, de la stratégie de

${ }^{26}$ A titre d'exemple, entre 1996 et 2002, plus de 7000 visas ont été attribués à des élèves de la minorité pour effectuer leur scolarité secondaire en Turquie, souvent assortis de bourses. Et 5000 autres visas pour des étudiants à l'Université, cela signifie que plus de 12000 élèves et étudiants ont quitté la Thrace durant ce laps de temps, soit plus de $10 \%$ de la population composant la 'minorité musulmane' estimée en Thrace! Ces chiffres (approximatifs) étaient manifestement plus importants dans les années 1980 ; ces informations nous ont été fournies sous couvert de confidentialité. 
Hersant, Jeanne (2008) 'Surveillances croisées et rivalité gréco-turque en Thrace occidentale : entre coercition et contrôle social', European Journal of Turkish Studies, Thematic Issue N 8 , No. 8 | Surveiller, normaliser, réprimer, URL : http://www.ejts.org/document2693.html

To quote a passage, use paragraph (§).

sensibilisation des instances européennes menées par les associations de Turcs de Thrace occidentale d'Allemagne (Hersant 2007). Kemal est parti travailler en Allemagne dans le cadre des accords d'envoi de main d'œuvre avec la Grèce, après avoir été expulsé de Turquie, ce qui lui a interdit l'accès à l'université où il s'était inscrit27. Aujourd'hui retraité à Komotini, il aimerait briguer un mandat local mais affirme n'avoir aucune chance tant qu'il ne sera pas 'réhabilité par le consulat'.

[21] Le consulat général de Turquie aurait ainsi eu le pouvoir de faire et défaire les réputations et les carrières politiques des candidats turcs, en fonction de leur allégeance ou au contraire de leur trahison supposée à la cause et à l'État turc. Étant donné ce climat de suspicion généralisée, les rumeurs constituaient en effet un mode de régulation sociale, empêchant la contestation, qui pouvait coûter cher aux personnes visées en termes de 'communiste' ou 'traître/agent des Grecs'. La liste noire aurait été progressivement supprimée à partir de 1992, après les élections législatives d'octobre 1991 en Turquie, et l'arrivée du gouvernement de Süleyman Demirel (Hersant 2007: 385). Le quadrillage de la population concernait également la prospection menée par les instituteurs auprès des bons élèves musulmans qui parlaient couramment le grec, susceptibles d'être envoyés à Chypre pour seconder l'administration turque :

J'étais très bon élève au lycée ${ }^{28}$ et l'un de mes professeurs, qui faisait partie du contingent d'enseignants envoyés par la Turquie, voulait absolument que je fasse des études, mais ma famille était très pauvre alors j'ai renoncé, il fallait gagner de l'argent. Avec un ami, on a posé notre candidature pour partir travailler en Allemagne, en 1970, deux mois après on a eu une réponse. Un jour j'ai été contacté par un fonctionnaire du consulat général de Turquie, c'était un ami de mon professeur, celui qui voulait que j'étudie. II m'a donné rendez-vous dans un parc, à Komotini, et m'a proposé de partir à Chypre comme espion. Ils faisaient ce genre de proposition aux bons élèves de la minorité, ceux qui parlaient le grec couramment. J'ai refusé, leurs histoires ne m'intéressent pas, de toute façon j'avais décidé de partir en Allemagne ${ }^{\prime 29}$.

[22] En somme c'est aussi sur le terrain de la délation, de la suspicion et de la surveillance, qu'ont été mis en place les mécanismes de la co-gouvernementalité, en instillant dans une

${ }^{27}$ Entretien réalisé en juillet 2005. Aujourd'hui encore, nombre de jeunes musulmans n'ont pas un niveau suffisant en grec pour passer le concours d'entrée à l'Université en Grèce, extrêmement sélectif, même pour les jeunes dont le grec est la langue maternelle. Depuis 1995 existe un quota de $0,5 \%$ de musulmans dans les universités, qui sont de facto dispensés de passer le concours.

${ }^{28}$ II s'agit du lycée Celal Bayar de Komotini.

${ }^{29}$ Entretien avec un ancien Gastarbeiter aujourd'hui au chômage, en février 2003. Voir aussi Papadakis (2005: 76). 
Hersant, Jeanne (2008) 'Surveillances croisées et rivalité gréco-turque en Thrace occidentale : entre coercition et contrôle social', European Journal of Turkish Studies, Thematic Issue N 8 , No. 8 | Surveiller, normaliser, réprimer, URL : http://www.ejts.org/document2693.html

To quote a passage, use paragraph (§).

population cette séparation en deux 'camps', la défiance vis-à-vis de l'autre ; en affirmant la sphère d'influence de chaque État par de telles loyautés. Ce sentiment demeure aujourd'hui, quoique de façon atténuée, en raison du droit de veto dont disposent de fait les acteurs du champ sécuritaire grec tout comme le consulat turc sur les candidatures turques lors des consultations électorales (Hersant, Yatropoulos 2008).

\section{Nouveaux modes de contrôle en temps de paix}

\section{Coercition aléatoire et tensions entre champs politique et sécuritaire grecs}

[23] Depuis le début des années 2000, la détente politique observée, en Thrace et dans les relations gréco-turques en général. L'ouverture politique est également liée au processus d'adaptation aux normes européennes en matière de respect des droits des minorités (Hersant 2000), et se traduit par un relâchement de la surveillance des autorités grecques sur la 'minorité musulmane'. La tutelle administrative sur la région et le rôle des institutions étatiques de sécurité nous invite pourtant à nuancer l'ouverture politique en Thrace occidentale (Hersant 2009), et la liberté de parole et d'action des acteurs politiques turcs, pourtant bien réelles depuis la fin des années 1990 (Hersant, Yatropoulos 2008). Ce sont en effet deux logiques qui s'affrontent: une logique politique et électorale d'ouverture, et une logique sécuritaire appuyée sur la raison d'État, selon laquelle les musulmans constituent une menace potentielle, particulièrement en raison de la tutelle du consulat général de Turquie sur une partie de cette population.

[24] Hormis l'encadrement de la désignation des candidats aux élections législatives, l'ouverture aux Turcs du champ politique local en Thrace est contrebalancée par les interdictions sporadiques qui frappent les manifestations culturelles turques, contre l'avis des responsables politiques grecs locaux. Un incident survenu en mai 2003 illustre les tensions entre l'ouverture du champ politique local d'une part, la permanence des institutions de sécurité et de la logique sécuritaire, de l'autre. À l'occasion d'un festival de printemps, Hıdrellez30, organisé dans le village de Thermes, le bus qui transportait le groupe de musique et danses folkloriques de l'Union turque de Xanthi est arraisonné à l'entrée du village par des barrages de police et ses occupants interdits de

${ }^{30}$ II s'agit d'une célébration héritée des pratiques du début du christianisme, traditionnellement honorée en Thrace par les musulmans bektachi en tant que 'fête du printemps' (Dalègre 1995), aujourd'hui réinvestie par les acteurs du mouvement identitaire turc. 
Hersant, Jeanne (2008) 'Surveillances croisées et rivalité gréco-turque en Thrace occidentale : entre coercition et contrôle social', European Journal of Turkish Studies, Thematic Issue N 8 , No. 8 | Surveiller, normaliser, réprimer, URL : http://www.ejts.org/document2693.html

To quote a passage, use paragraph (§).

festivités. Le commandement en chef du quatrième corps d'armée, le chef de la préfecture de police de Xanthi et le secrétaire général de la région Macédoine orientale-Thrace occidentale ont avancé l'argument suivant : l'autobus transportait dans la soute à bagages des pancartes en turc (Hıdrellez bayramınız kutlu olsun) 31 .

[25] Apprenant l'incident, le préfet élu du département de Xanthi, M. Pavlidis, téléphone immédiatement au président de l'Union turque de Xanthi, qui se trouvait dans le bus, pour lui assurer qu'il n'est pour rien dans ces événements, et qu'il ne peut rien faire en raison de sa position dans la hiérarchie des pouvoirs locaux ${ }^{32}$. Le député Galip Galip interpelle le gouvernement à ce sujet, de son côté le Comité de surveillance Helsinki-Grèce condamne l'événement au titre de la violation de la liberté de circulation et d'expression. Les faits, ainsi que la protestation du Comité de Surveillance Helsinki, sont relatés dans le journal de gauche $A v g^{\beta 3}$. L'année suivante, le festival a eu lieu sans incident. Cet épisode illustre la permanence du rôle prééminent des institutions de sécurité dans la hiérarchie des pouvoirs locaux, comme l'exprime le dépit de l'élu apportant son soutien au président de l'Union turque.

[26] Cette tension s'exprime à travers la réaffirmation en ces circonstances de l'identité turque de la 'minorité musulmane', dans la mesure où nombre d'élus locaux s'estiment lésés dans leurs prérogatives par le maintien des institutions de sécurité. C'est en cela qu'on ne peut réellement parler d'articulation des champs politique et sécuritaire, comme cela peut être le cas en Turquie où l'imbrication de ces champs est caractéristique du régime :

L'originalité du régime sécuritaire tient à la relation entre le politique institutionnel (présidence, Parlement, partis politiques) et le champ sécuritaire, c'est-à-dire le réseau d'institutions liées à la sécurité : armée en premier lieu, mais aussi police, services secrets, diplomatie, justice (Dorronsoro 2005 : 22).

31 || s'agit d'une formule rituelle pour se souhaiter une joyeuse fête. 'Iskeçe'de Türkçe yasağı' [Interdiction du turc à Xanthi], Cumhuriyet, 08.05.03 ; 'AB'de Jivkov rüzgarı' [Comme un air de Jivkov dans l'Union européenne], Gündem, 09.05.03. Jivkov est le nom du dirigeant bulgare qui en 1989 a appelé, lors d'une allocution télévisée, 'ceux qui ne se sentent pas bulgares' à quitter le pays, donnant ainsi le coup d'envoi de l'expulsion massive des Turcs.

32 Ibid. Sur l'organisation administrative de la Grèce et le statut particulier de la région Macédoine orientale-Thrace, voir (Hersant 2008).

33 'Ilıca konusunda yazılı soru' [Questions écrites au sujet de Thermes], Gündem, 16.05.03 ; 'llıca yasaǧına yankılar sürüyor !' [Les échos à l'interdiction de Thermes continuent !], Gündem, 23.05.03. La Grèce exerçait alors la présidence tournante de l'Union européenne. 
Hersant, Jeanne (2008) 'Surveillances croisées et rivalité gréco-turque en Thrace occidentale : entre coercition et contrôle social', European Journal of Turkish Studies, Thematic Issue N 8 , No. 8 | Surveiller, normaliser, réprimer, URL : http://www.ejts.org/document2693.html

To quote a passage, use paragraph (§).

[27] Les acteurs appartenant à chacun de ces champs sont en concurrence dans la gestion de la question minoritaire. Les uns ont une approche sécuritaire de cette question, les autres souhaitent intégrer l'électorat musulman à la vie politique locale. Or les institutions tutélaires ont un poids non négligeable dans le champ politique local, notamment dans la désignation des candidats turcs aux élections législatives. L'ouverture politique est donc contrebalancée par la possibilité que se réservent les autorités militaires grecques d'intervenir à tout moment pour rappeler aux musulmans leur altérité. II s'agit d'une politique d'intimidation jouant sur l'arbitraire : interdire tantôt ce qui a été auparavant autorisé, ou vice-versa ; ainsi, l'année précédente comme l'année suivante, en période électorale, tous les candidats turcs ont tenu meeting en turc (Hersant, Yatropoulos 2008).

[28] On sait par ailleurs le rôle du folklore dans la construction des identités régionales et ethniques, à l'instar de ce qu'il est à la construction nationale (Öztürkmen 1998); les manifestations folkloriques sont justement le terrain d'expression symbolique de cet arbitraire, mais aussi des tensions entre ouverture du champ politique et logique sécuritaire. Ainsi, alors que les élèves des 'écoles de la minorité' sont associés comme les autres enfants aux fêtes nationales grecques, les équipes de folklore des associations turques se voient refuser le droit de participer aux commémorations. En mai 2004, à l'occasion des festivités commémorant la libération de la Thrace en 1944, le groupe folklorique de la mairie d'Edirne (ville turque frontalière de la Thrace) a été invité par la mairie de Komotini à se produire sur la grand place ; en revanche la demande de l'Association des membres de la minorité musulmane diplômés de l'Université de présenter son groupe folklorique dans le cadre des célébrations officielles est restée sans suite ${ }^{34}$.

\section{Normaliser plutôt que réprimer : entre diplomatie et notabilité locale}

[29] La détente politique se traduit également par un changement des modalités de prise en charge de la 'minorité musulmane', par le consulat général de Turquie mais aussi par les autorités grecques : depuis 2000, un diplomate est en poste à Xanthi, le responsable du Bureau des Affaires politiques de la minorité, représentation locale du ministère des Affaires étrangères auparavant basée à Kavala. L'intérêt des diplomates grecs et turcs en poste dans la région pour les villages, particulièrement ceux du nord de Xanthi, labellisés comme étant 'pomaques', est un phénomène

34 “'Trakya'nın Kurtuluşu' kutlamaları başlıyor' [Les cérémonies de commémoration de la libération de la Thrace commencent], Gündem, 07.05.04. 
Hersant, Jeanne (2008) 'Surveillances croisées et rivalité gréco-turque en Thrace occidentale : entre coercition et contrôle social', European Journal of Turkish Studies, Thematic Issue N 8 , No. 8 | Surveiller, normaliser, réprimer, URL : http://www.ejts.org/document2693.html

To quote a passage, use paragraph (§).

récent, lié à la pacification du contentieux et au déplacement de l'objet de ce contentieux. Côté turc, l'objectif est de décloisonner l'espace, d'unifier les représentations et le sentiment d'appartenance à la turcité. Côté grec, la démarche est à l'inverse de faire en sorte que les habitants des zones rurales locuteurs d'un bulgare dialectal osent s'affirmer comme 'Pomaques'. Dans la mesure où il n'y a plus de conflit ouvert entre les deux pays, l'affrontement symbolique entre les intérêts nationaux grecs et turcs se vit sur le mode de la délimitation de zones d'influence micro locales. Les villages de montagne situés dans l'ancienne zone sous contrôle militaire, dont certains sont reliés par une route asphaltée au chef-lieu de leur département depuis dix ans à peine, constituent aujourd'hui une des scènes de la rivalité gréco-turque. Les institutions civiles représentant les deux États ne se donnent plus à voir comme des instruments de coercition mais comme des intermédiaires, à travers la façon dont la personnalité qui les incarne investit un rôle nouveau : 'Ce sont des investissements, collectifs ou individuels, épisodiques ou continus, qui "donnent corps" à une institution et la font exister socialement' (Lagroye 2006 : 16-17).

[30] Avec l'installation à Xanthi du Bureau des affaires politiques de la minorité, le représentant de l'Etat grec est entré dans le rôle d'un notable local, se montrant préoccupé non plus de sécurité mais du bien-être de ses concitoyens, et occupant une partie de son temps à des visites de courtoisie dans les villages. II a à cœur d'expliquer aux habitants qu'ils ne doivent pas avoir peur de se dire Pomaques (et non Turcs), et qu'ils 'doivent prendre conscience de leur statut de citoyens européens, dans un État fier de s'être adapté aux normes européennes ${ }^{135}$. De ce point de vue, le diplomate grec joue son rôle de notable qui va au devant de ses administrés et leur sert d'intermédiaire ou de 'personne ressource', leur expliquant le fonctionnement de l'État et les droits dont ils bénéficient. L'anecdote que me rapporta le responsable du Bureau des affaires culturelles est éclairante de ce point de vue.

Alors qu'il effectuait une visite dans un village de l'ancienne zone militaire fermée et s'était attablé dans un café, un homme est venu le trouver, catastrophé : il avait perdu son passeport alors qu'il devait s'envoler le lendemain en Allemagne comme travailleur saisonnier. Le responsable du Bureau des affaires culturelles lui expliqua qu'il lui suffisait de se munir de sa carte d'identité puisqu'en vertu des accords de Schengen il pouvait circuler librement dans l'espace européen. Mais son interlocuteur refusait de le

${ }^{35}$ Entretien réalisé en août 2002. 
Hersant, Jeanne (2008) 'Surveillances croisées et rivalité gréco-turque en Thrace occidentale : entre coercition et contrôle social', European Journal of Turkish Studies, Thematic Issue $N^{\circ} 8$, No. 8 | Surveiller, normaliser, réprimer, URL : http://www.ejts.org/document2693.html

To quote a passage, use paragraph (§).

croire : il ne pouvait imaginer que désormais les choses soient si simples ${ }^{36}$. Jouant son rôle d'intermédiaire jusqu'au bout, le diplomate s'engagea à aller avec lui le lendemain jusqu'à l'aéroport de Thessalonique pour lui prouver qu'il disait vrai. L'homme dubitatif accepta tout de même son explication, et à son retour trois mois plus tard il se rendit au Bureau des affaires culturelles pour remercier le diplomate qui l'avait si bien tiré d'affaire ${ }^{37}$.

[31] Tout autant que les prérogatives juridiques définies dans le traité de Lausanne, par ailleurs en grande partie obsolète (Hersant 2007), le consul général de Turquie est quant à lui celui qui fait vivre la diplomatie et le nationalisme turc en Thrace. Jusqu'aux années 1980, il a cantonné ses activités aux zones urbaines de Komotini et Xanthi, ne s'intéressant aux populations villageoises, et a fortiori 'pomaques', que depuis que celles-ci constituent un enjeu dans la définition des intérêts nationaux grecs et turcs concurrents dans la région. Les mémoires d'un ambassadeur turc en poste à Athènes en 1973 (Gürün 1994), qui fut le second diplomate turc à être autorisé à se rendre en Thrace occidentale, sont éclairantes: elles illustrent une prise de conscience de la formation 'archaïque' du personnel religieux, et de l'absence dans les villages d'enseignants formés aux valeurs de la République turque. Pour autant, les consuls alors en poste ne s'intéressaient manifestement pas à l'arrière-pays. Adil Özgüç, un instituteur envoyé en Thrace par le gouvernement turc dans le cadre de l'Accord culturel passé en 1968 entre les deux Etats, déplorait en 1974 que le rôle du consul ne se limite qu'à s'insérer dans les réseaux de sociabilité des notables locaux :

Les responsables du consulat se sont toujours abstenus de contacts avec le peuple [...].Pourtant la minorité turque ne se compose pas seulement du groupe des ağa et notables. Lorsque l'on sort du centre-ville pour aller dans les villages, le véritable visage de la minorité apparaît. [...] Pour une partie des fonctionnaires du consulat, Komotini vit dans le dénuement. Parce qu'ici il n'y a pas le style de vie des villes européennes auxquelles ils sont habitués. [...] Ils passent la première partie de leur temps à s'habituer à l'endroit, et la seconde partie à se préparer à leur prochain poste (Özgüç 1974 : 168).

[32] Aujourd'hui la turcité ne se réduit plus à une lutte idéologique entre partisans et opposants de la Turquie républicaine ; la turcité étant autant un signe de différenciation sociale qu'un marqueur politique (Hersant 2009a), le consul général de Turquie affirme dans ses pratiques sa posture de notable. Ce constat ouvre de nouvelles perspectives dans la mesure où la sociologie

${ }^{36}$ Jusqu'aux années 1990, les habitants de la zone sous contrôle militaire devaient avoir un laissezpasser en leur possession.

${ }^{37}$ Entretien réalisé en août 2002 
Hersant, Jeanne (2008) 'Surveillances croisées et rivalité gréco-turque en Thrace occidentale : entre coercition et contrôle social', European Journal of Turkish Studies, Thematic Issue $\mathrm{N}^{\circ} 8$, No. 8 | Surveiller, normaliser, réprimer, URL : http://www.ejts.org/document2693.html

To quote a passage, use paragraph (§).

politique française a tendance à considérer la figure du notable principalement sous les traits d'un élu local. Par ailleurs, la zone militaire fermée a été démantelée entre 1996 et 2002, il n'est plus considéré comme un signe d'hostilité que le consul général de Turquie s'y rende. Le notable, d'après les définitions conventionnelles, est un pourvoyeur de ressources (Massicard 2004), un intermédiaire entre la population et l'administration centrale vis-à-vis de laquelle il bénéficie néanmoins d'une autonomie d'action (Grémion 1976: 249) ${ }^{38}$. Julien Fretel (2004: 46) souligne également sa 'propension à constituer des groupes sociaux réunis autour de causes non politiques mais susceptibles néanmoins de peser sur les enjeux électoraux et partisans'. Adopter cette posture de notable soucieux du bien-être de ses 'coreligionnaires' (dindaş, selon le terme diplomatiquement en usage $)^{39}$ permet justement de dépolitiser, ne serait-ce qu'en apparence, la question de la turcité. Nous ajouterons à cela le fait que la notabilité implique des pratiques sociales distinctives, et le fait de cultiver 'l'entre-soi'.

\section{Délimiter l'espace social de la turcité}

[33] En tant que notable, le consul général de Turquie adopte le comportement de la bourgeoisie urbaine et parle avec une relative condescendance de la population locale : les membres de la minorité sont selon lui des gens incultes (cahil) qui ont toujours besoin qu'on leur montre l'exemple avant de faire quelque chose ${ }^{40}$. D'où le rôle de la Turquie, à travers sa représentation consulaire; en somme le paternalisme est souvent de mise dans les rapports sociaux. Les réceptions 'privées' du consul relatées il y a presque 40 ans, sont très proches de celles qu'il donne aujourd'hui. Ainsi, en octobre 1967, 'à l'occasion de son départ', le consul de l'époque 'a donné un

${ }^{38}$ Cette autonomie d'action, certes relative dans le cas d'un diplomate, s'est illustrée pendant la campagne pour les élections législatives de 2004 : alors que le gouvernement turcs soutenait le candidat de la Nouvelle Démocratie et actuel Premier ministre, le consul général en poste à Komotini soutenait un notable local - le député sortant du PASOK - contre le candidat local de la Nouvelle Démocratie, qui était un inconnu.

${ }^{39}$ Le terme turc 'soydaş' qu'on pourrait traduire à la fois par 'compatriote' et 'congénère' (de même ascendance, à la différence de 'concitoyen', plus neutre) a été à l'origine d'une rupture diplomatique en 1991. Lors d'affrontements entre musulmans et chrétiens en Thrace, à l'occasion desquels le consul turc avait exprimé sa solidarité non pour ses 'coreligionnaires' mais pour ses 'compatriotes' ; il a été reconduit à la frontière, mais pas désavoué par le gouvernement turc de l'époque.

${ }^{40}$ Entretien avec un ancien consul, juin 2003. 
Hersant, Jeanne (2008) 'Surveillances croisées et rivalité gréco-turque en Thrace occidentale : entre coercition et contrôle social', European Journal of Turkish Studies, Thematic Issue $\mathrm{N}^{\circ} 8$, No. 8 | Surveiller, normaliser, réprimer, URL : http://www.ejts.org/document2693.html

To quote a passage, use paragraph (§).

dîner d'adieu pour les notables'41. En septembre 2004 le rituel est assez semblable, si ce n'est que le consul avait organisé en outre une cérémonie publique d'adieux rassemblant une centaine de personnes, dont les élus et dirigeants grecs locaux ${ }^{42}$. Hormis l'entretien de rapports avec les autres notables turcs, son rôle social est en grande partie tourné vers les zones rurales et les élus locaux ('grecs' et 'turcs'); le quadrillage de la population revient désormais à 'occuper le terrain' concurremment avec son homologue grec du Bureau des affaires politiques. II a cependant un avantage sur ce dernier, qui en tant que représentant de l'Etat et incarnation de la tutelle politique sur la région, reste à l'écart des réseaux d'élus et des rituels politiques locaux.

[34] Le consul ne se contente plus aujourd'hui d'activités mondaines. Le quota d'instituteurs négocié avec l'État grec ${ }^{43}$ depuis 1968, ne permet plus d'envoyer des enseignants que dans les lycées de la minorité des villes de Komotini et Xanthi. La présence turque dans les zones rurales s'affirme désormais par la mise à disposition de prêcheurs par la Diyanet $t^{44}$ pendant les fêtes religieuses (Hersant 2007), d'une part; et d'autre part par les déplacements du consul dans les villages, à l'occasion de telle ou telle activité folklorique organisée par les associations culturelles turques. Ces visites sont autant d'occasions de visites de courtoisie aux élus municipaux et de bains de foule. Au même titre que les candidats lors des divers élections (Hersant, Yatropoulos 2008), le consul s'efforce désormais de s'impliquer dans la vie sociale locale. II se passe rarement une semaine sans que le journal Gündem relate l'une de ses visites dans tel ou tel village, notamment dans l'ancienne zone militaire fermée au nord de Xanthi.

[35] Le consul en poste de 2001 à 2004 a joué un rôle pionnier en allant visiter des villages où jamais aucun de ses prédécesseurs ne s'était rendu, par exemple le village de Thermes, aux

${ }^{41}$ C'est-à-dire 'les anciens députés et les notables de la cemaat, ainsi que les journalistes turcs'. 'Gümülcine Konsolosumuz değişti' [Notre consul à Komotini a changé], Batı Trakya, Octobre 1967, 7, p. 17.

42 'Botsalı, Türk ve Yunan dostlarıyla vedalaştı' [Botsalı a fait ses adieux à ses amis grecs et turcs], Gündem, 03.09.04.

${ }^{43}$ En fonction du nombre d'élèves dans les écoles dites minoritaires dans chacun des deux États, négociation d'autant plus difficile qu'il ne reste quasiment plus d'élèves dans les écoles grecques d'Istanbul (Anastassiadou, Dumont 2003).

${ }^{44}$ Direction des Affaires religieuses en Turquie. 
Hersant, Jeanne (2008) 'Surveillances croisées et rivalité gréco-turque en Thrace occidentale : entre coercition et contrôle social', European Journal of Turkish Studies, Thematic Issue N 8 , No. 8 | Surveiller, normaliser, réprimer, URL : http://www.ejts.org/document2693.html

To quote a passage, use paragraph (§).

confins du département de Xanthi, à quelques kilomètres de la frontière bulgare ${ }^{45}$. Puis en juin 2004, quelques semaines avant son départ, le consul a entrepris une tournée d'envergure dans les villages 'pomaques' de l'ancienne zone militaire fermée afin d'assister aux réjouissances de fin d'année organisées dans les différents cours de broderie et tissage par l'Union turque de Xanthi : il a visité treize villages en une semaine ${ }^{46}$. Ces 'visites de courtoisie' (nezaket ziyaretleri) ${ }^{47}$ ne s'effectuent jamais hors de la présence d'un des journalistes de Gündem, et de plusieurs notables locaux : édiles et député turcs, présidents d'associations turques, etc. Représentant d'une Turquie laïque longtemps hostile aux institutions religieuses locales ${ }^{48}$, le consul va même désormais jusqu'à participer à certaines cérémonies religieuses dans les villages, prenant la parole à la mosquée pour déclarer à quel point 'il est heureux de se joindre au peuple pour de telles cérémonies traditionnelles' ${ }^{49}$. Le consul de Turquie multiplie les apparitions publiques et privées : son statut de notable se caractérise justement par le fait que la frontière est ténue entre les deux. Ainsi, lorsque ni le consul ni son suppléant ne se rendent à un mariage, leur absence est considérée comme un affront et revêt une signification politique: c'est une façon d'adouber ou au contraire de renier les dirigeants ou prétendants à la direction des organisations turques, d'Allemagne ou de Thrace (Hersant 2007). Le consul est aussi un pourvoyeur de ressources en termes de rémunérations et de visas (supra), mais

45 'llica'da Başkonsolos'a sevgi şöleni' [Une cérémonie chaleureuse en l'honneur du consul général à Thermes (llica)], Gündem, 26.09.03

${ }^{46}$ 'Erken yaz ziyaretleri' [Visites estivales anticipées], Gündem, 18.06.04.

${ }^{47}$ C'est le terme employé dans le journal Gündem pour décrire les visites dont doit s'acquitter le nouveau consul à son arrivée en Thrace. 'Ba konsolos Demirer İTB'yi ziyaret etti' [Le consul général Demirer a rendu visite à l'Union turque de Xanthi], Gündem, 29.09.06. On notera que ce terme, en référence aux pratiques mondaines de savoir-vivre dont la courtoisie est le mode de régulation (voir sur ce point Pinçon, Pinçon-Charlot 1989), a vocation à dépolitiser les visites du consul.

${ }^{48}$ Les importantes prérogatives du mufti, notamment en matière de juridiction civile, telles qu'accordées par le traité de Lausanne alors que la Turquie moderne s'est constituée notamment sur la base de l'inféodation des structures religieuses à l'État et de la sécularisation de la société, expliquent l'absence de relations entre le consul et le personnel religieux de la Thrace jusqu'aux années 1970.

${ }^{49}$ 'Yassı̈̈ren halkından dayanışma örneǧi' [Un exemple de solidarité donné par le peuple d'Oreon (Yassı̈̈ren)], Gündem, 25.04.03. 
Hersant, Jeanne (2008) 'Surveillances croisées et rivalité gréco-turque en Thrace occidentale : entre coercition et contrôle social', European Journal of Turkish Studies, Thematic Issue N 8 , No. 8 | Surveiller, normaliser, réprimer, URL : http://www.ejts.org/document2693.html

To quote a passage, use paragraph (§).

aussi en ce qu'il promeut les activités de communication des membres du mouvement identitaire turc, devenus ces dernières années militants et experts de leur cause (Hersant 2007) ${ }^{50}$.

[36] La présence systématique du consul lors des événements culturels locaux semble par ailleurs constituer en soi un registre d'action du champ politique local. Durant la période au cours de laquelle a été dépouillé le journal Gündem, il n'est pas d'événement concernant la 'minorité musulmane' - de la pose de la première pierre lors des travaux d'agrandissement du lycée Celal Bayar de Komotini à l'inauguration d'un cours de tissage de tapis dans un village de montagne ${ }^{51}$ - qui ne rassemble le consul de Turquie ou le vice-consul, ainsi que des élus grecs locaux. Souriant aux côtés des élus et acteurs associatifs turcs, ils offrent ainsi un tableau de 'l'amitié gréco-turque', dans une sorte de rituel devenu immuable. La reconnaissance et la bonne entente réciproque de tous ces acteurs semblent conforter chacun d'entre eux dans sa position de notable. Les commémorations et célébrations officielles organisées par le consulat en présence des notables turcs constituent aujourd'hui autant de rituels d'allégeance à la République turque, destinés à la 'minorité musulmane'. Elles constituent également des moments de mise en visibilité de la co-gouvernementalité dans la mesure où les personnalités grecques locales participent à ces cérémonies : les élus 'grecs' locaux sont systématiquement invités aux manifestations nationales turques et aux manifestations folkloriques turques.

[36] Pour autant, de tels rituels reviennent en certaines circonstances à faire des règles de la bienséance le marqueur de l'altérité. Les personnalités politiques grecques ne sont jamais que les convives de ces manifestations, elles semblent n'y être jamais associées, et ce statut leur est parfois rappelé de façon peu amène. C'est notamment le cas pendant les campagnes pour les élections

${ }^{50}$ Comme par exemple la traduction en turc et la diffusion, en 2003, d'un 'Guide des minorités aux Nations Unies'. L'activité de L'Association des diplômés de la minorité consiste à fournir des rapports 'clé en main' au Conseil de l'Europe, qui évalue chaque année la situation des minorités dans plusieurs pays. Lors d'un entretien avec la consul turc en 2003, celui-ci nous a donné un 'rapport européen' censé nous servir à 'comprendre la situation', qui était en fait le même que celui que nous avaient remis précédemment un représentant de l'Association.

51 'Celal Bayar Lisesi için tarihî bir gün' [Un jour historique pour le lycée Celal Bayar], Gündem, 24.09.02 ; 'Yassı̈̈ren'de kilim ve halı dokuma kursu' [Cours de tissage de tapis à Oreon (Yassıören)], Gündem, 08.10.02. 
Hersant, Jeanne (2008) 'Surveillances croisées et rivalité gréco-turque en Thrace occidentale : entre coercition et contrôle social', European Journal of Turkish Studies, Thematic Issue N 8 , No. 8 | Surveiller, normaliser, réprimer, URL : http://www.ejts.org/document2693.html

To quote a passage, use paragraph (§).

locales, au cours desquelles tous les candidats 'grecs' ${ }^{152}$ investissent les associations turques. En 2002, à l'occasion d'une 'soirée de solidarité' organisée par l'Association des Diplômés de la Minorité (Batı Trakya Azınlık Yüksek Tashilliler Derneği), à laquelle assistaient la plupart des candidats 'grecs', le maire de Komotini subit une humiliation : il ne put pas faire un discours comme on le lui avait promis ${ }^{53}$. Le camouflet fut d'autant plus rude que le consul turc avait, lui, fait un discours.

\section{Conclusion}

[37] La surveillance de la population musulmane Thrace occidentale et la mise sous tutelle de la région reposent toujours, côté grec, sur un dispositif de sécurité ; néanmoins ce dispositif est atténué par l'abandon des pratiques coercitives au profit de pratiques 'incitatives' de la part des institutions représentant localement les deux Etats. La notion de co-gouvernementalité s'avère féconde en ce qu'elle permet de revisiter la notion de dispositifs de sécurité, et l'usage différencié qui peut en être fait par un Etat selon qu'il s'agit d'affirmer son contrôle sur son territoire, ou sur la population d'un autre territoire que le sien. La transformation du rôle du consul turc est une manifestation de cette co-gouvernementalité, quand celui-ci cesse d'être simplement le représentant d'une institution consulaire pour devenir celui qui incarne cette institution, et prendre le rôle d'un notable impliqué dans la vie sociale du groupe sur lequel s'exerce sa tutelle. II faut en somme considérer non seulement l'aspect politique et administratif des prérogatives déléguées à cet acteur étatique, mais aussi sa stature de notable local.

[38] L'analogie peut être faite avec l'étude que fait Jacques Lagroye de la 'formalisation ou mise en forme de l'institution' présidentielle en France après 1962 (Lagroye 1992 : 162). Le consulat général de Turquie peut être qualifié d'institution politique, 'au sens le plus simple de cadre organisé de la vie politique officielle' (Lacroix, Lagroye 1992 : 8) ; le rôle du consul quant à lui relève d'une forme d'institution corollaire, de la même façon qu'en France, justement en raison de la polémique

${ }^{52}$ Pour les élections locales les candidats n'ont pas l'obligation d'être membres d'un parti ; en général les listes constituées sont soutenues par l'un des deux grands partis, voire par une coalition (comme ce fut le cas en 2002 avec l'alliance entre le PASOK et le Synaspismos).

53 'BTAYTD'den yardımlaşma gecesi' [Le gala de solidarité de l'Association des diplômés (ici désignée par son acronyme)], Gündem, 24.09.02. 
Hersant, Jeanne (2008) 'Surveillances croisées et rivalité gréco-turque en Thrace occidentale : entre coercition et contrôle social', European Journal of Turkish Studies, Thematic Issue N 8 , No. 8 | Surveiller, normaliser, réprimer, URL : http://www.ejts.org/document2693.html

To quote a passage, use paragraph (§).

relative à l'élection au suffrage universe $\left.\right|^{54}$, le rôle du Président de la République s'autonomise en quelque sorte de la fonction définie dans la Constitution de 1958. Le point commun entre ces deux cas de figure est le fait que l'on est face à deux institutions qui existent à travers un unique rôle. Ainsi, l'institution présidentielle en France est définie juridiquement autour de la fonction du président de la République, qui dans le cadre de cette fonction assume un rôle politique, social, mais aussi symbolique. Dans le cas du consulat général de Turquie en Thrace occidentale, l'institution ne se réduit pas en théorie à la fonction du consul : elle n'est pas personnifiée comme peut l'être la présidence de la République où l'institution équivaut à la fonction. En pratique pourtant, le rôle social, politique et symbolique du consul aujourd'hui a fini par transcender l'institution.

${ }^{54}$ Le conflit portait d'une part sur l'élection du Président de la République au suffrage universel direct, d'autre part sur les modalités de la réforme qui a été qualifié de 'coup de force constitutionnel' par le président du Sénat de l'époque, Gaston Monerville. 
Hersant, Jeanne (2008) 'Surveillances croisées et rivalité gréco-turque en Thrace occidentale : entre coercition et contrôle social', European Journal of Turkish Studies, Thematic Issue $N^{\circ} 8$, No. 8 | Surveiller, normaliser, réprimer, URL : http://www.ejts.org/document2693.html

To quote a passage, use paragraph (§).

\section{References}

Aarbakke, Vemund (2000) The Muslim Minority of Greek Thrace, thèse de doctorat, Université de Bergen, Norvège.

Anastassiadou, Meropi (1991) 'Trois livres sur les Pomaks de Grèce', Lettre d'information du groupe de travail sur la transmission du savoir dans le monde musulman périphérique, n¹1, p. 64-66.

Anastassiadou, Méropi ; Dumont, Paul (2003) 'Une mémoire pour la Ville : la communauté grecque d'Istanbul en 2003', Les Dossiers de l'IFEA, Institut français d'études anatoliennes.

Bertrandn Gilles (2004) Le conflit héléno-turc, Paris, Maisonneuve et Larose.

Clayer, Nathalie (2007) Aux origines du nationalisme albanais, Paris, Karthala.

Dalègre, Joëlle (1995) 'E.C. Zenginis: Calendrier des fêtes et culte des saints en Thrace occidentale', Lettre d'information sur le monde musulman périphérique, n¹5, p. 55- 58.

Dalègre, Joëlle (1997) La Thrace grecque. Population et territoires, Paris, L'Harmattan.

Foucault, Michel (2001) Dits et écrits II, 1976-1988, Paris, Gallimard.

Foucault, Michel (2004) Sécurité, territoire, population. Cours au Collège de France. 1977-1978, Paris, Seuil/Gallimard.

Fretel, Julien (2004) 'Le parti comme fabrique de notables. Réflexion sur les pratiques notabiliaires des élus de l'UDF', Politix. Trajectoires de la notabilité, I. Pratiques et stratégies, vol. 65, p. 45-72.

Grémion, Pierre (1976) Le pouvoir périphérique : bureaucrates et notables dans le système politique français, Paris, Seuil.

Gürün, Kâmuran (1994) Bükreş-Paris-Atina. Büyükelçilik anıları [Bucarest-Athènes-Paris, souvenirs d'ambassades], stanbul, Milliyet Yayınları.

Hersant, Jeanne (2007) 'Mobilisations politiques, co-gouvernementalité, construction ethnique. Sociologie du nationalisme turc à travers le cas des Turcs de Thrace occidentale (Grèce, Allemagne, Turquie)', thèse de doctorat en sociologie sous la direction de Hamit Bozarslan, EHESS, Paris.

Hersant, Jeanne (2009) 'Contourner les normes européennes grâce... aux instruments européens. L'impératif de sécurité nationale ou les résistances à l'intégration européenne de la Grèce' in Costa, Olivier ; Roger, Antoine ; Saurugger, Sabine (dir.) 'Les résistances à l'intégration européenne', Revue Internationale de Politique Comparée, 16 (1), à paraître.

Hersant, Jeanne (2009a) 'La politique d'accueil des "étrangers d'ascendance turque" en Turquie : le statut dérogatoire des réfugiés apatrides en provenance de Thrace occidentale (Grèce)', Revue Européenne des Migrations Internationales, 24 (3), à paraître.

Hersant, Jeanne ; Yatropoulos, Nepheli (2008) 'Mobilisation identitaire et représentation politique des "Turcs" en Thrace grecque : les élections législatives de mars 2004', European Journal of Turkish Studies, Articles, URL : http://www.ejts.org/document1342.html

Lacroix, Bernard ; Lagroye, Jacques (dir.) (1992) Le Président de la République, usages et genèse d'une institution, Paris, Presses de la FNSP. 
Hersant, Jeanne (2008) 'Surveillances croisées et rivalité gréco-turque en Thrace occidentale : entre coercition et contrôle social', European Journal of Turkish Studies, Thematic Issue $\mathrm{N}^{\circ} 8$, No. 8 | Surveiller, normaliser, réprimer, URL : http://www.ejts.org/document2693.html

To quote a passage, use paragraph (§).

Lagroye, Jacques (1992) 'Le conflit de l'automne 1962. Dispersions et convergences dans la formalisation du rôle' in Lacroix, Bernard ; Lagroye, Jacques (dir.) Le Président de la République, usages et genèse d'une institution, Paris, Presses de la FNSP, p. 161-193.

Lagroye, Jacques (2002) 'L'institution en pratiques', Revue Suisse de Science Politique. À propos de Pierre Bourdieu, vol. 8, n 3-4, p. 129-155.

Lagroye, Jacques (2006) La vérité dans l'Eglise catholique. Contestations et restauration d'un régime d'autorité, Paris, Belin.

Leveau, Rémy (1991) 'Les partis politiques et l'intégration des "beurs"' in Mény, Yves (dir.) Idéologies, partis politiques et groupes sociaux. Études en l'honneur de Georges Lavau, Paris, Presses de la FNSP, p. 229-243.

Massicard, Elise (2004) 'Entre l'intermédiaire et l'homme d'honneur. Savoir-faire et dilemmes notabiliaires en Turquie', Politix. Trajectoires de la notabilité, II., vol. 67, p. 101-127.

Özgüç, Adil (1974) Batı Trakya Türkleri [Les Turcs de Thrace occidentale], Istanbul, Kutlu Yayınları.

Papadakis, Yiannis (2005) Echoes From the Dead Zone. Across the Cyprus Divide, London/NewYork, I.B. Tauris \& Co.

Pinçon, Michel ; Pinçon-Charlot, Monique (1989) Dans les beaux quartiers, Paris, Seuil. 